\title{
CHRONOLOGIE DE L'APPORT AZOTÉ PENDANT LE CYCLE DE REPRODUCTION CHEZ LA TRUIE
}

\author{
P. H. DUEE \\ avec la collaboration technique de Michèle SERFzat, Christiane Vachot et J. Lrbost \\ Station de Recherches sur l'Elevage des Porcs, \\ Centre national de Recherches zootechniques, I. N.R.A., \\ 78350 Jouy en Josas
}

\section{RÉSUMÉ}

Une expérience a été entreprise durant 2 cycles de reproduction sur 76 truies nullipares de race Large White réparties en 3 groupes. A un lot témoin (lot I), dont le taux azoté du régime est faible en gestation (ro p. Ioo) puis élcvé en lactation (I6 p. Ioo), on compare d'une part un groupe d'animaux recevant un régime à taux azoté constant ( 13 p. Ioo) pendant tout le cycle de reproduction (lot 3). D'autre part, on expérimente (lot 2) la possibilité d'apporter un complément azoté en fin de gestation (Io p. Ioo puis 13 p. Ioo à partir du $75^{\mathrm{e}}$ jour de gestation) tout en réduisant 1'apport de lactation ( 13 p. Ioo). Les matières azotées du régime sont apportées sous forme de blé remplacé partiellement ou non par le tourteau de soja.

En gestation, l'augmentation de $30 \mathrm{p}$. Ioo de la consommation de matières azotées accroît le bénéfice pondéral des truies, comme leur bilan azoté : l'élévation du gain net de gestation est de 32 p. Ioo (I er cycle) ou de 66 p. Ioo ( $2^{e}$ cycle). L'apport azoté de gestation n'influence pas la taille de la portée à la mise bas, ou au sevrage ( 35 jours), ni le poids moyen des porcelets à la naissance.

Durant la première lactation, la croissance des porcelets sous la mère est comparable dans les 3 lots mais la diminution de consommation azotée en lactation (lots 2 et 3 ) accroît la perte de poids chez la mère : $17,7 \mathrm{~kg}(\operatorname{lot} 2)$ et $\mathbf{I}_{5}, 0$ (lot 3 ) contre $4,8 \mathrm{~kg}$ (lot $\mathbf{1}$ ). Durant la seconde lactation, une diminution du gain de portée apparaît dans le lot $3(45,15 \mathrm{~kg}$ contre 49, $5 \mathrm{~kg}$ dans le lot I) et surtout dans le lot $2\left(3^{8,8} \mathrm{~kg}\right)$.

Par ailleurs, les valeurs énergétiques de la matière sèche du lait, déterminées à I 5 jours de lactation, sont plus élevées dans le lot i que dans les deux autres lots. L'influence respective des taux azotés de gestation et de lactation est discutée dans ce mémoire.

\section{INTRODUC'TION}

De nombreux travaux, résumés récemment (RÉRAT et DUÉE, I975) montrent la possibilité de réduire considérablement l'apport azoté durant le cycle de reproduction chez la Truie. En gestation, le niveau de consommation azotée semble ne 
pas influencer les performances de reproduction à la mise bas (PoND it al., I968; De GEETER et al., I972). En lactation, un bilan azoté nul est obtenu, chez la Truie adulte avec un apport protéique d'environ 700 grammes par jour (ÉTIENNF et al., I975).

Il se peut, cependant, qu'une carence partielle en certains acides aminés indispensables durant la gestation modifie la lactogénèse (I)UÉE et RÉRAT, I975). Le problème des apports optima de matières azotées, en gestation et en lactation et des relations existant entre eux se pose alors. C'est dans ce but que l'on a conduit la présente étude. A un lot témoin, dont le taux azoté du régime est faible en gestation puis élevé en lactation, on a comparé d'une part un groupe d'animaux recevant un régime à taux constant de matières azotées durant tout le cycle de reproduction. D'autre part, on a expérimenté la possibilité d'apporter un complément azoté en fin de gestation, tout en réduisant l'apport de lactation. Compte tenu des effets différents qui peuvent être obtenus sur truies nullipares ou sur truies adultes, l'étude a été poursuivie sur 2 cycles.

\section{MATÉRIEL ET MÉTHODES}

L'expérience a été entreprise sur 76 animaux de race Lavge White, d'âge et de poids moyens à la puberté, respectivement de $23 \mathrm{I}$ jours et de $10_{4} \mathrm{~kg}$. Eintre le premicr et le second cestrus, les animaux, maintenus dans les mêmes conditions, ont une croissance journalière de $+37 \pm 21$ grammes calculée sur une durée moyenne de $21,0 \pm 0,3$ jours. A la saillie, qui intervient donc au second estrus détecté, les animaux sont répartis en trois groupes ne différant que par la chronologie de l'apport de matières azotées durant le cycle de reproduction (tabl. I). Les animaux des lots $\mathrm{I}$ et 3 reçoivent, durant le cycle de reproduction, approximativement la même quantité de protéines, distribuée de façons différentes : si, dans le lot 3 , le régime distribué (régime B) est identique en gestation comme en lactation, l'apport journalier de matières azotées dans le lot I est, par contre, plus faible en gestation (régime A), mais plus élevé en lactation (régime C).Les animaux du lot 2 reçoivent le même régime que les animaux du Jot 1 pendant les 75 premiers jours de gestation (régime A), puis le même régime que les animaux du lot 3 (régime B) pendant le reste du cyle. Durant l'intervalle sevrage-saillie, les animaux reçoivent le même régime (régime $B$ ).

TABLEAU I

Schéma expérimental ( ${ }^{(1)}$

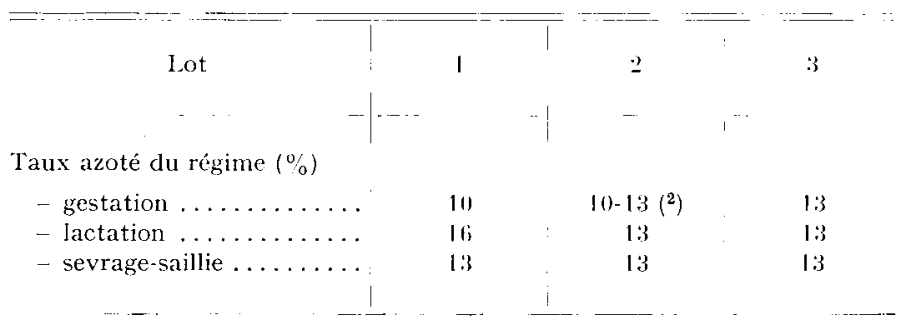

(1) Effectif initial d'animaux : 76 ; expérience poursuivic pendant deux cycles de reproduction.

(2) Changement dc régime au $75^{\mathrm{e}}$ jour de gestation. 
Les régimes expérimentaux ne diffèrent que par leur teneur en matières azotées. La source azotée est constituée de blé, remplacé partiellement ou non par le tourteau de soja 5o (tabl. 2). Les régimes sont enrichis en énergie, par l'incorporation de $3 \mathrm{p}$. Ioo d'huile d'arachide et en cellulose, par l'adjonction de cellulose de bois à des taux différents permettant ainsi un apport similaire d'énergie digestible : un essai en cages de digestibilité a d'ailleurs montré que les teneurs en énergie digestible étaient, en moyenne, de $3220 \mathrm{kcal} / \mathrm{kg}$

\section{TABLEAU 2}

Composition et caractéristiques des régimes

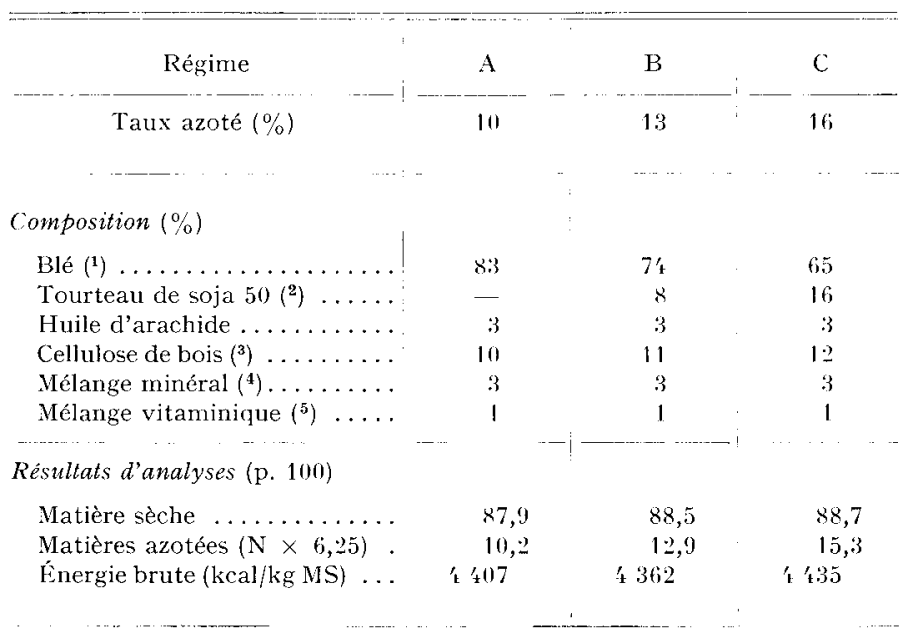

(1) Blé à 85,6 p. 100 de matière sèche et 1',3 p. 100 de matières azotées par rapport à la matière sèche durant la première gestation; par la suite, à 86,8 p. 100 de matière sèche et 13,2 p. 100 de matières azotées par rapport à la matière sèche.

(2) Tourteau à 88,4 p. 100 de matière sèche, renfermant ${ }^{2} 9,1$ p. 100 de matières azotées.

$\left(^{3}\right)$ Cellulose purifiée extraite du bois à $9 \geq, 7$ p. 100 de matière sèche et 87,3 p. 100 de cellulose par rapport à la matière sèche.

(4) Composition du mélange minéral (en p. 100 de la ration) : phosphate bicalcique : 1,2 ; craie broyée : 1,2 ; chlorure de sodium : 0,5 ; oligoéléments : 0,1 .

(5) Mélange vitaminique dont la composition est rapportée par DUéE et Rérat (1975).

En gestation, le niveau alimentaire quotidien est fixé à $2 \mathbf{k g}$, en lactation, à $4,5 \mathrm{~kg}$ ( $\mathrm{I}$ er cycle) ou $5,5 \mathrm{~kg}$ ( $2 \mathrm{e}$ cycle) et durant l'intervalle sevrage-saillie, à $2,5 \mathrm{~kg}$.

Durant cette étude qui se déroule sur deux cycles de reproduction, les variations pondérales des mères sont enregistrées ainsi que leurs performances de reproduction à la mise bas et au sevrage ( 35 jours). Vers le $82^{\mathrm{e}}$ jour de la première gestation, Io animaux de chaque lot sont soumis, après une semaine d'adaptation, à une période de 5 jours en cages de digestibilité pendant laquelle on récolte séparément les fèces et l'urine dans le but de déterminer la digestibilité apparente des matières azotées ingérées et la rétention d'azote. Sur 6 d'entre eux on renouvelle cette étude au même stade physiologique au cours du second cycle. Enfin, à r 5 jours de lactation et sur l'ensemble des animaux, on effectue un prélèvement de lait, représentatif d'une têtée, dont on détermine la composition globale (matière sèche, cendres, azote, lactose, énergie). L'analyse statistique des résultats est effectuée au moyen du test d'analyse de variance. Les animaux reve- 
nant en chaleurs post-coïtum étant éliminés de l'étude, cette analyse ne porte que sur 63 truies en première gestation et sur $4^{8}$ truies en seconde gestation. De l'effectif initial, 59 p. Ioo des truies sèvrent leur seconde portée, sans que la nature du régime distribué ait un effet sensible sur le taux d'élimination.

\section{RÉSULTATS}

\section{I. - Variations pondérales des truies au cours de l'expérience}

L'augmentation de 30 p. Ioo de la consommation de matières azotées en gestation (lot 3) accroît significativement le bénéfice pondéral des truies aux deux cycles de l'étude (tabl. 3) : l'élévation đu gain net de gestation est de 32 p. Ioo au premier cycle et de $66 \mathrm{p}$. Ioo au second cycle. L'accroissement de consommation de matières azotées durant le dernier tiers de la gestation (lot 2 ) élève également le gain de poids des animaux, d'une manière significative ( $\mathrm{I}^{\mathrm{er}}$ cycle) ou non ( $2^{\mathbf{e}}$ cycle).

\section{TABLEAU 3}

Performances de reproduction des animaux

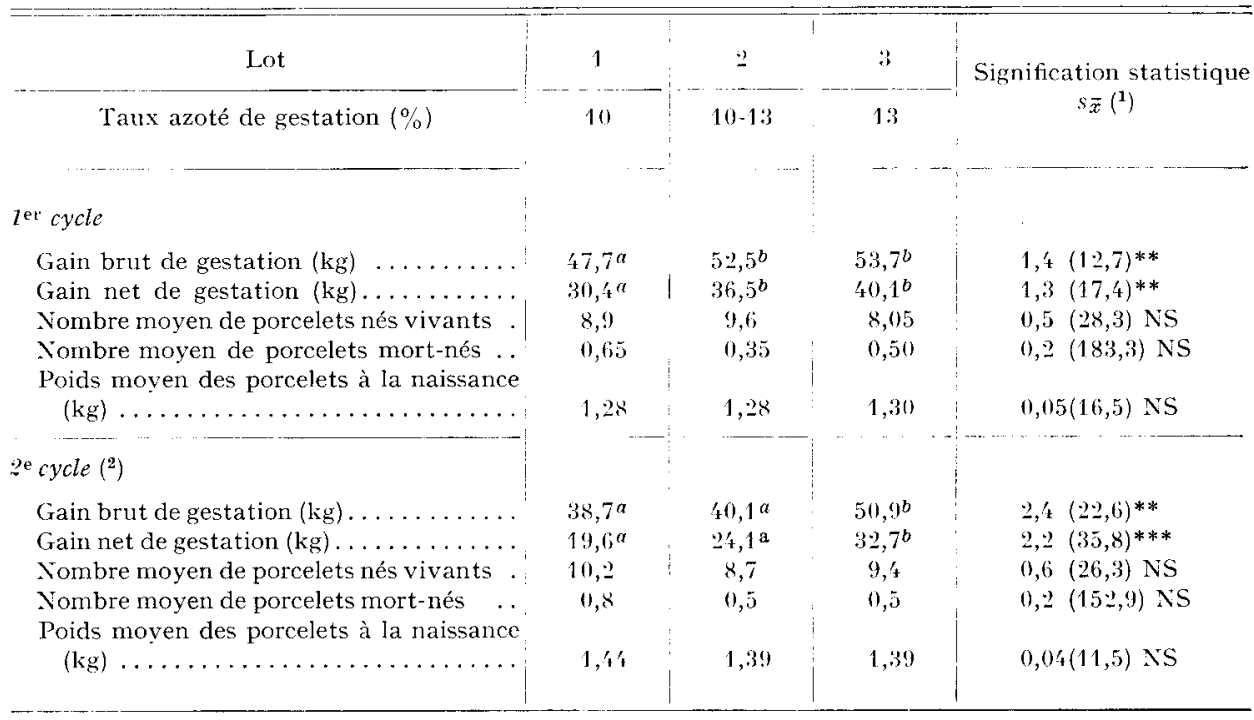

(1) Écart-type de la moyenne (coefficient de variation). NS : différence non significative au seuil $\mathrm{P}=0,05 ; *$ : différence significative au seuil $\mathrm{P}=0,05 ; * *$ : différence significative au seuil $\mathrm{P}=0,01 ; * * *$ : différence significative au seuil $\mathrm{P}=0,001$.

Les valeurs suivies de la même lettre ne sont significativement pas différentes.

(2) Diminution significative du gain brut de gestation $(P<0,001)$ et du gain net de gestation $(\mathrm{P}<0,001)$, augmentation significative du poids moyen des porcelets à la naissance $(\mathrm{P}<0,01)$, au cours du second cycle. 


\section{TABLEAU 4}

Performances des truies au cours de la lactation

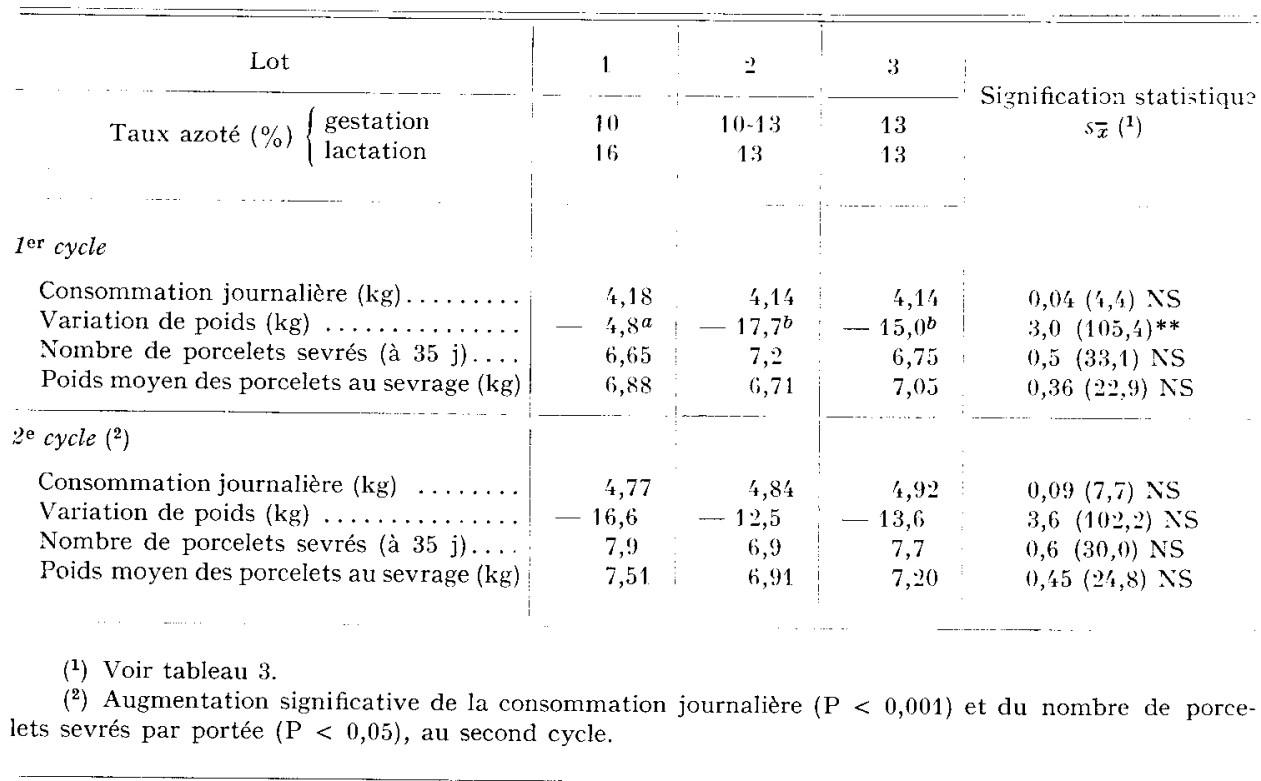

\section{TABLEAU 5}

Intervalle sevrage-premier cestrus : durée et variations pondérales des truies

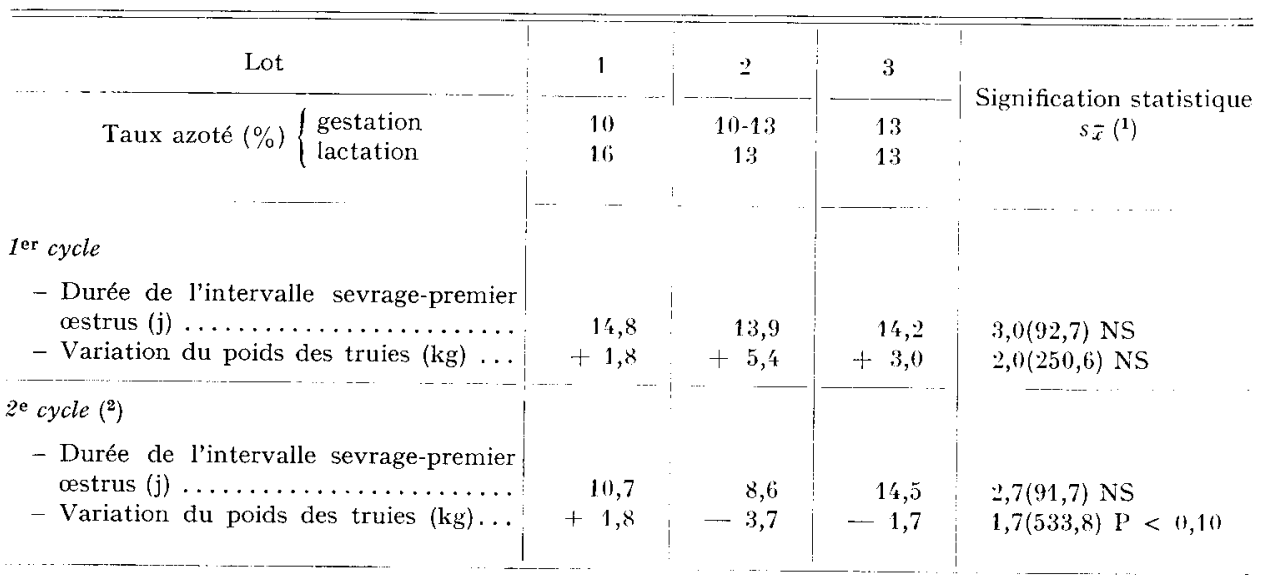

(1) Voir tableau 3.

(2) Diminution significative de la variation du poids des truies $(\mathrm{P}<0,01)$, après le second sevrage. 
tu cours de la première lactation, les animaux perdent significativement plus de poids quand ils reçoivent le régime le plus bas en azote ( $\operatorname{lots} 2$ et 3 ). La différence entre les 3 lots n'apparaît plus au second cycle : la perte pondérale moyenne s'établissant à $\mathrm{I} 4,3 \mathrm{~kg}$ (tabl. 4). Il est à noter que la consommation des mères allaitantes n'est pas affectée par le taux azoté du régime, aux deux cycles de l'expérience.

Après le sevrage et jusqu'au premier ostrus détecté, les animaux reçoivent le même régime (à 13 p. roo de matières azotées) et les variations pondérales ainsi que la durée de cette période sont conparables dans les trois lots, au cours du premier cycle (tabl. 5). Par contre, au cours du second cycle, les truies des lots 2 et 3 perdent du poids, au contraire des animaux du lot I. Chez les animaux ayant achevé deux cycles de reproduction (soit un effectif de 48 truies) on a pu comparer les variations pondérales durant chacun des cycles. Il s'avère qu'au cours du second cycle de reproduction, les truies, alimentées dans les mênes conditions qu'au premier cycle, présentent en gestation une croissance plus faible : le gain net diminue en effet de 29 p. Ioo. En lactation, si la perte de poids est plus importante seulement chez les aninatux du lot $\mathrm{I}$, la consommation est, par contre, plus élevée pour l'ensemble des truies. Enfin, après le sevrage et jusqu'à la saillie, le poids des truies diminue dans les lots 2 et 3 .

\section{II. - Performances de reproduction à la mise bas ct au sevrage}

Il ne semble pas que la quantité de matières azotées consonmées par la mère au cours de la gestation influe sur la taille de la portée à la mise bas ainsi que sur le poids moyen des porcelets (tab1. 3). Cependant, le nombre de porcelets nés vivants des truies ayant terminé les deux premières gestations apparaît plus élevé dans les lots I et 2 (I 8,7 et I8,6 respectivement) dont les animaux reçoivent le taux azoté le plus bas durant les deux premiers tiers de la gestation, mais l'augmentation moyenne d'un porcelet par rapport au lot 3 n'est pas significative. Cette tendance disparaît d'ailleurs au sevrage où le nombre de porcelets sevrés par les truies ayant réalisé deux lactations est, en moyenne, de I4,2.

Le poids moyen des porcelets sevrés est peu affecté par le taux azoté du régime maternel (tabl. 4). Au second cycle, pourtant, une tendance à la diminution du poids des porcelets au sevrage apparait dans le lot 3 et surtout dans le lot 2 : ce ralentissement de la croissance des porcelets sous la mère s'accentue principalement à partir du $2 I^{\mathrm{e}}$ jour de lactation, sans que la consommation des porcelets durant cette phase ne soit modifiée (tabl. 6).

Il s'ensuit que le gain de portée, qui est peu affecté par le taux azoté du régime maternel avant ou après la mise bas, au premier cycle, est significativement plus éleré au second cycle dans le lot I ( I 0 p. Ioo de matières azotées) que dans le lot 2 (I3 p. IOo) ; les truies du lot 3 présentent, pour leur part, un gain de portée intermédiaire. Comme précédemment, on a pu établir des différences significatives entre les performances réalisées dans chacun des cycles. C'est ainsi que le nombre de porcelets sevrés est plus élevé au second cycle, ce qui, en grande partie, explique l'augmentation du gain de portée.

La croissance des porcelets a été suivie après leur sevrage et jusqu'à l'âge de 2 mois. Les porcelets issus des mères du lot 2 présentent le gain de poids le plus faible, aux 2 cycles de l'étude, mais cette diminution de croissance n'est pas signifi- 
cative ( 8 p. Ioo au premier cycle, 4 p. roo au second cycle). I1 semble surtout que la mortalité durant cette période soit plus élevée dans le lot 2 : le pourcentage de pertes augmente de $6,2 \mathrm{p}$. IoO (lot I) à I7,5 (lot 2) pour les porcelets du premier cycle, et de 7,3 p. Ioo à 16,3 pour ceux du second cycle. L,es pertes enregistrées dans le lot 3 durant cette période sont voisines de celles du lot $\mathrm{I}$, soit respectivement Io,9 et 8,9 p. Ioo.

\section{TABIEAU 6}

Croissance et consommation des porcelcts sous la mère

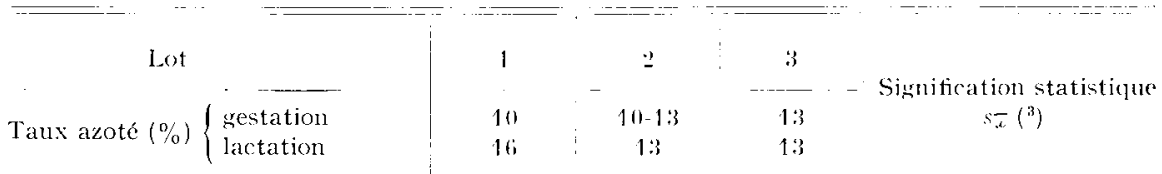

ler $y c l e$ (kg/période)

- Croissance moyennc

$$
14-21 \text { jours }
$$

$21-35$ jours

3,32

3,10

3,18

$(1,20)(25,2) \quad N S$

Consommation $21-3 ;$ jours $\left({ }^{2}\right) \ldots \ldots \ldots$

$\stackrel{2}{2}-28$

$2,11: 2$

2,28

$0,21(11,1)$ NS

11,33

0,18

0,53

$0,15(110,6)$ NS

(1)-21 jours

$2-2, \geq 01$

$2.5,16$

13,48

23,30

1 ' $t, 1$

39,314

37,77

$1,8: 3(3: 3,7) \times \mathrm{NS}$

1, 1 ( $13,3,3) \times S$

$11-35$ jours $\ldots \ldots \ldots \ldots \ldots \ldots \ldots \ldots$; $31,1,1 ;$

2e cycle (3) (kg/période)

- Croissance moyeme

$11-21$ jours $\ldots \ldots \ldots \ldots \ldots \ldots \ldots \ldots$
$\geq 1-3,3$ jours $\ldots \ldots \ldots \ldots \ldots \ldots$

$: 3,1 ; 7$

$\because, 7+2$

$3, \therefore, 10$

$3,6,1$

$11,21(2: 3,7)$ NS

Consonmation $(2) \geq 1-35$ jours

0,59

$1,1 \mathrm{~N}$

$\stackrel{2}{2}, \underline{6}$

$0,25(1,6,6) \times S$

Gain de portée

$11-21$ jours

$\because 1-35$ jours

30,27

11,34

0,$6 ; 6$

$0,10(97,1) \mathrm{NS}$

$11-35$ jours

$\div, 72$

$13,11^{b}$

29,08

$16,118^{a b}$

$2,23(31,4)$ NS

$3 \times, 8 \div$

45,16

$1,1,8(1,9)$ *

$3,38(30,5) \mathrm{P}<0,10$

(1) Voir tableau :3.

(2) La consommation par porcelet, de 11 ì 21 jours, est en moyennc de 7:2 grammes au premier cycle et de 87 grammes au second cycle et n'est pas affectée par le taux azoté du régime maternel.

(3) Le gain de portée ( 1 à 21 jours et $0-35$ jours) augmente significativement $\left(\mathrm{P}^{3}<0,01\right)$ au 2 e cycle.

\section{III. - C'tilisations digestive et métabolique de l'azote ingéré}

L'étude en cages à métabolisme réalisée vers $1 \mathrm{e} 82^{\mathrm{e}}$ jour de gestation porte sur un effectif important d'animaux par lot, correspondant à $4^{8} \mathrm{p}$. Ioo de l'effectif total au premier cycle et 40 p. Ioo au second cycle.

L'accroissement de la consommation de matières azotées par la Truie (lot 3) a pour première conséquence d'augmenter l'excrétion d'azote fécal (tabl. 7) mais l'utilisation digestive apparente de l'azote ingéré, qui ne tient pas compte 
TABLEAU 7

Utilisation digestive apparente de l'azote ingévé en gestation (')

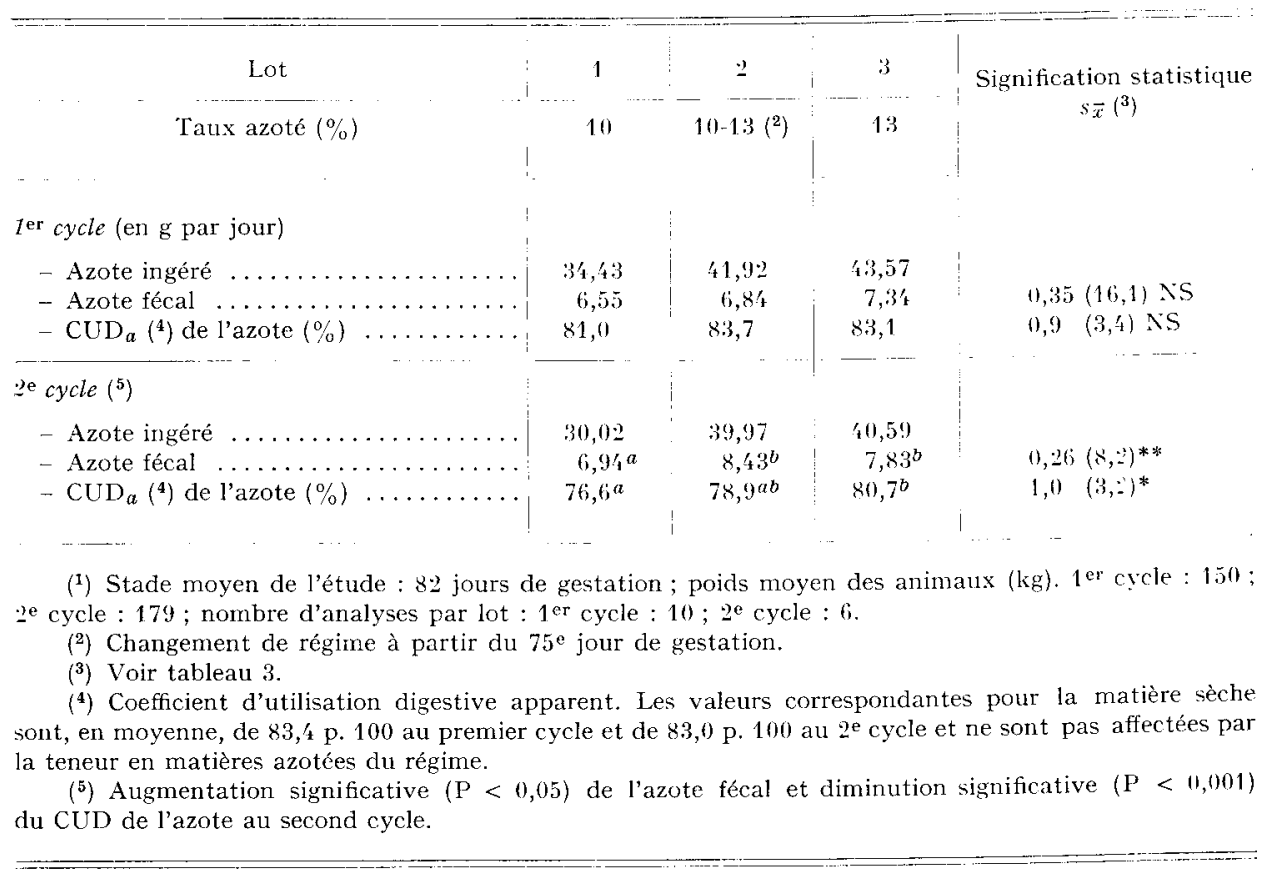

\section{TABLEAU 8}

Utilisation métabolique de l'azote absorbé en gestation (1)

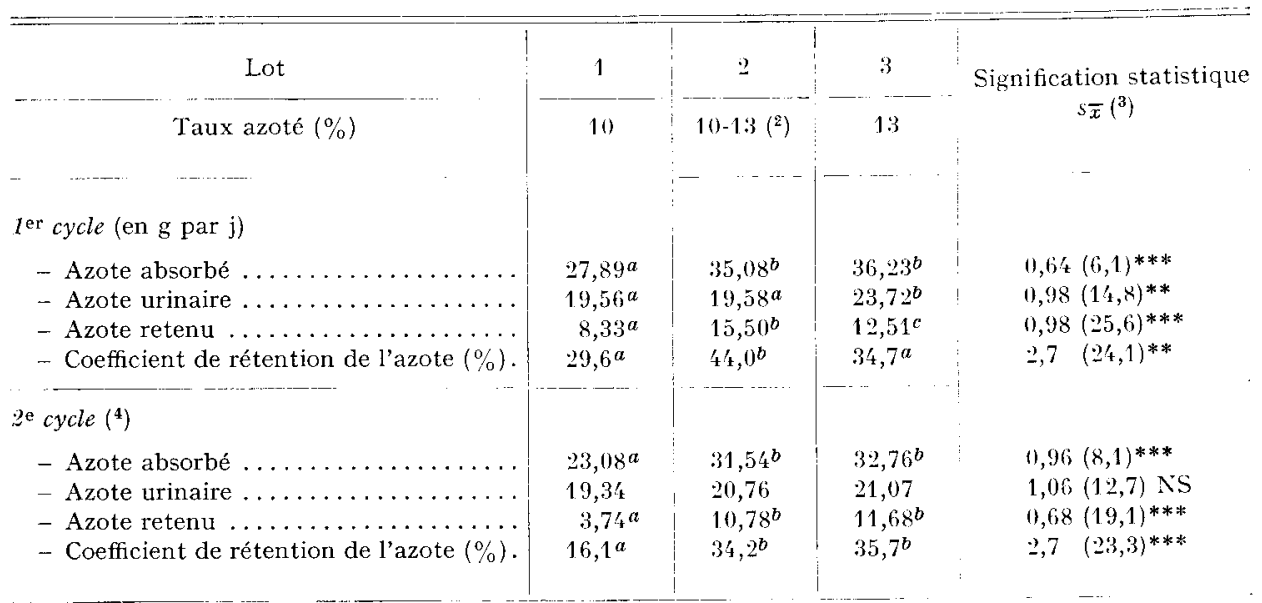

$\left({ }^{1}\right),\left({ }^{2}\right),\left({ }^{3}\right)$ Voir tableau précédent.

(4) Diminution significative $(\mathrm{P}<0,05)$ de l'azote retenu et du coefficient de rétention de l'azote au second cycle. 
des pertes endogènes, semble augmenter au taux azoté le plus élevé. Cette amélioration atteint d'ailleurs le seuil de signification au second cycle de reproduction. Il s'ensuit que l'azote absorbé par l'animal est plus élevé dans le lot 3 . Cette augmentation de l'azote absorbé accroît significativement les pertes azotées urinaires au premier cycle : le bilan azoté est cependant amélioré aux taux azoté le plus haut, sans que cela traduise une amélioration de l'utilisation métabolique de l'azote absorbé (tab1. 8). Au second cycle, par contre, le coefficient de rétention de l'azote est significativement accru dans le lot 3, compte tenu de pertes azotées urinaires comparables dans les lots I et 3 .

La réalisation de bilans azotés sur les animaux du lot 2 pose le problème du temps d'adaptation au régime dans la mesure où les collectes de fèces et d'urine sont effectuées peu après le changement de régime, vers le $75^{\circ}$ jour de gestation. Ainsi, les pertes azotées fécales et urinaires au premier cycle sont similaires à celles enregistrées sur les animaux du lot I, ce qui peut surestimer la quantité d'azote réellement retenue. Au second cycle, pourtant, le temps d'adaptation semble suffisant et les pertes fécales et urinaires comme le bilan azoté sont comparables à ceux des animaux du lot 3. Au cours de cette étude, on a pu également mettre en évidence une diminution de la digestibilité apparente des matières azotées de 4,5 points quand l'animal est plus âgé. Par ailleurs, le bilan azoté ainsi que le coefficient de rétention de l'azote semblent moins élevés au second cycle, ce que l'on peut relier à la diminution de l'azote absorbé.

\section{IV. - Composition du lait à 15 jours" de lactation}

La composition du lait des truies a été effectuée à I 5 jours de lactation et l'ensemble des résultats est rapporté dans le tableau 9 . Cette étude porte sur 95 analyses dont 52 durant le premier cycle de reproduction. Les teneurs moyennes exprimées en grammes pour Ioo $\mathrm{ml}$ sont, pour la matière sèche, les matières minérales, l'azote et le lactose, respectivement de I $8,2 \mathrm{I}, 0,843,0,785$ et 5,64. Par rapport à la matière sèche du lait, ces teneurs s'établissent respectivement à 4,65, 4,32 et 3 r,43 pour roo gammes, pour une valeur énergétique moyenne de 6 I 35 calories par gramme.

Aucune différence liée à l'apport de protéines en gestation comme en lactation n'est statistiquement significative au seuil étudié $(\mathrm{P}=0,05)$. I1 s'avère, cependant, qu'au cours des 2 cycles de reproduction, les teneurs en matière sèche du lait sont plus importantes dans le lot I, qui correspond à l'apport le plus large de protéines en lactation. Parallèlement, les valeurs énergétiques de la matière sèche du lait sont plus élevées dans le lot $\mathbf{I}$, cette augmentation étant plus sensible au second cycle. Inversement, les concentrations en lactose, par rapport à la matière sèche, augmentent dans les deux lots où l'apport de protéines est réduit en lactation. Enfin, les teneurs en matières minérales ou en matières azotées ne sont pas affectées par la nature du régime.

La comparaison des teneurs en matière sèche du lait et de la composition de la matière sèche, au cours des deux cycles, a été effectuée à partir de 39 couples de données. Les différences les plus importantes portent sur la teneur en matière sèche qui diminue au second cycle, et surtout en lactose qui augmente significativement. 
TABLEAU 9

Composition du lait à 15 jours de lactation

Nombre d'analyses : ${ }^{\text {er }}$ cycle : $5^{2} ; 2^{\text {e }}$ cycle : 43

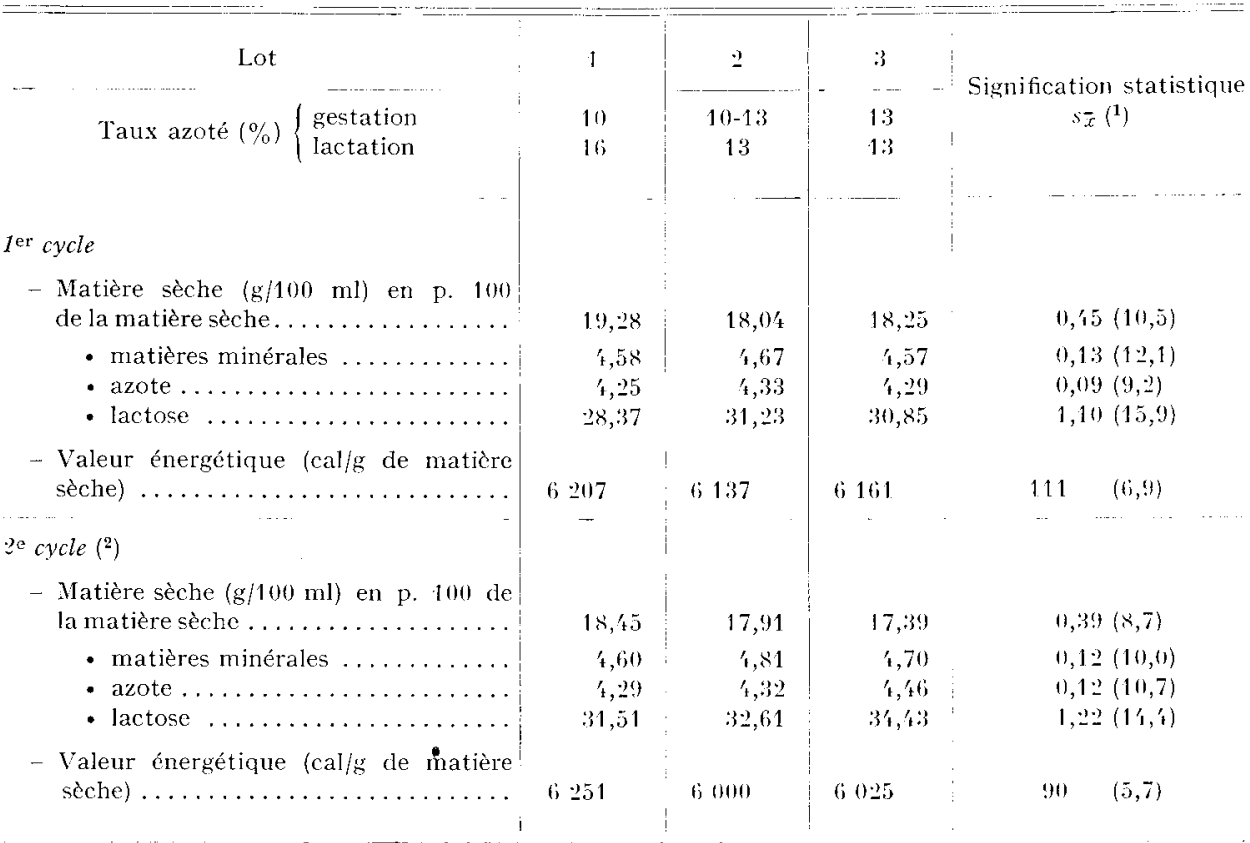

(1) Écart-type de la moyenne (coefficient de variation). Aucune différence n'est statistiquement significative au seuil $\mathrm{P}=0,05$.

(2) Différence statistiquement significative entre cycles : matière sèche $(P<0,10)$; lactose $(P<0,01)$.

\section{DISCUSSION}

Les performances zootechniques des animaux enregistrées sur 2 cycles de reproduction semblent bien différenciées. D'une part, le bénéfice pondéral des truies au cours de la gestation est moins élevé quand la Truie est plus âgée. Cette diminution de croissance s'explique en partie par une diminution de consommation de matières azotées (note I du tab1. 2), associée à une digestibilité apparente plus faible. Cependant, les quantités d'azote excrété dans l'urine sont comparables d'un cycle à l'autre et il s'ensuit une diminution de l'azote retenu au second cycle, marquée surtout dans le lot I. Cette différence de réponse chez la Truie adulte par rapport à la capacité de rétention de la jeune Truie, en croissance encore active, a déjà été signalée par RoMbaUts (1962) et ErLsLEy et al. (1969). Il se peut pourtant que, dans le cas présent, l'apport énergétique ( $6440 \mathrm{kcal}$ d'énergie digestible par jour), légèrement inférieur aux normes du N.R.C. (I973), soit limitant, ce qui accuserait la diminution de l'azote retenu, dans le lot I (55 p. Ioo) où l'apport qualitatif de pro- 
téines est probablement limitant plus que dans le lot 3 (7 p. Ioo). D'autre part, le nombre de porcelets allaités et le gain de portée sont plus élevés au second cycle. Cette augmentation des dépenses de production en lactation est connue (SALMONLEGAGNEUR et al., I966). Elle souligne le fait que les besoins quantitatifs (azote, énergie) d'une truie adulte peuvent être différents de ceux d'une truie nullipare pendant cette phase du cycle, ce qui justifie la modulation de ces besoins suivant le poids de l'animal (A.R.C., I967; N.R.C., I973).

\section{I. - Effet du taux azoté en gestation}

L'accroissement du taux azoté de ro à I3 p. Ioo augmente, d'une façon significative, la vitesse de croissance des animaux au cours de la gestation, ce qui confirme les résultats de RipPel et al. (I965), Holden et al. (I968), Hawton et MEAdE (I97I), Mahax et Mangan (I975). L'augmentation du gain total ou du gain net de gestation se produit aux 2 cycles de l'étude, comme l'ont rapporté précédemment HoLDEN ct al. (I 968), HAW'TON et MEADE (I97I) mais non RiPPEL et al. (I965). Elle correspond, en partie, à une augmentation de l'azote retenu qui, en fin de gestation, s'élève en moyenne à $6 \mathrm{~g}$ d'azote par jour, valeur légèrement plus élevée que celle établie par Milier et al. (Ig69). I1 faut souligner cependant qu'un large apport de protéines en gestation ( I 7 à $20 \mathrm{p}$. IO0) n'améliore plus le bénéfice pondéral des truies ou la rétention azotée (Holden et al., I968 ; Mrller et al., I969; Mahan et Mangan, I975). MiLLER et al. (I969), pour leur part, estiment la quantité de matières azotées nécessaire pour une rétention azotée maximale en fin de gestation chez la Truie entre 285 et 342 grammes par jour. A la mise bas, les performances de reproduction sont comparables suivant les lots : ceci rejoint les conclusions de nombreux expérimentateurs incapables de mettre en évidence un effet du taux azoté de gestation sur la taille de la portée à la mise bas, même quand l'apport azoté est très faible (Pond et al., I968; De GEETER et al., I972; RIPPEL et al., I965). Le poids moyen des porcelets à la naissance peut, dans certains cas, subir le contrecoup d'une consommation faible de protéines par la mère (POND et al., I968) ou d'une carence partielle en certains acides aminés indispensables (DUÉE et RÉRAT, r975).

Dans ces conditions, l'accroissement de la consommation azotée durant le dernier tiers de la gestation (lot 2) profite essentiellement à la mère dont le gain net et la rétention azotée en fin de gestation augmentent, dans des proportions moins importantes cependant que celles rapportées par BAKER et al. (I97O).

Fn résumé, si aux consommations marginales de protéines ou de certains acides aminés indispensables, il peut exister une compétition entre la croissance maternelle et le développement fœtal, ce n'est plus le cas quand l'apport de matières

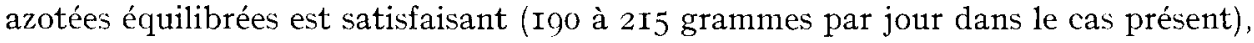
un apport complémentaire est alors bénéfique uniquement à la mère.

\section{II. - Apport azoté en lactation : infuence du taux azoté de gestation}

La restriction azotée appliquée au cours de la lactation correspond à un abaissement de la consommation de matières azotées de 640 grammes par jour à 535 au premier cycle et de 730 grammes à 630 au second cycle. Il est à noter que 
cette restriction ne modifie en rien le niveau de consommation des animaux, contrairement aux résultats de MAHAN et Grifo (I975) et l'on met donc en évidence l'effet propre de la restriction azotée. La comparaison des résultats reste toutefois limitée, compte tenu d'un apport azoté différent en gestation. Cependant, la restriction azotée en lactation a pour premier effet d'accroitre la perte de poids de lactation mais uniquesent au premier cycle, ce qui a déjà été souligné par McPhERSON et al. (Ig69) et par O'GRADY (I97I). Si les performances de reproduction au moment du sevrage sont peu affectées par le taux azoté de lactation, ce qui confirme des résultats antérieurs (McPherson et al., I969; O'Grady, I97I), il n'en est pas moins certain que le gain de portée qui traduit les potentialités laitières de la Truie, dépend en grande partie du passé nutritionnel de l'animal.

Pendant les trois premières semaines de lactation, la restriction azotée n'a pas d'effet sur le gain de portée au premier cycle de reproduction, quel que soit le niveau de rétention azotée durant la gestation précédente. Il est probable que cette absence d'effet soit la conséquence d'une participation plus importante, à ce stade, des réserves de gestation dans la synthèse des constituants du lait (SALMON-L_EGAGNEUR, I965; MAHAN et al., I97 I a). A I 5 jours de lactation, la composition du lait n'est que peu modifiée (augmentation de 5 p. Ioo de la teneur en matières azotées avec le taux azoté du régime maternel), il semble que seuls les taux de matière sèche et de lipides soient susceptibles d'être modifiés ce qui confirme les données de MAHAN et al. (I97I b) mais non celles d'ElLio'T' et al. (I97I).

De tels résultats se retrouvent, dans une certaine mesure, au cours du second cycle, bien que l'apport azoté complémentaire de fin de gestation ne permette plus de pallier la diminution du taux azoté de lactation. Il est vrai que les dépenses de production sont alors plus importantes (ce que traduit l'augmentation de la perte de poids dans le lot I, malgré l'accroissement de consommation) et, compte tenu de nos résultats (1)UéE et al., I975), le régime blé-soja à I3 p. Ioo de matières azotées est déficient principalement en lysine au cours de la lactation.

Après 21 jours de lactation, la croissance des porcelets ou le gain de portée diminue faiblement au premier cycle (lot 2), la diminution étant masquée par une augmentation de la consommation des porcelets (lot 3), comme le rapportent également McPherson et al. (I969). Au second cycle, par contre, les différences dans les gains de portée apparaissent plus importantes entre le lot à i 6 p. Ioo de protéines et les deux lots à I 3 p. Ioo, et sont d'autant plus accentuées que le gain net des truies durant la gestation est faible. Ceci souligne l'existence d'une relation entre le niveau des réserves accumulées en gestation et, d'une part, les performances laitières des truies, estimées dans cette expérience par le gain de portée et, d'autre part, la possibilité d'une réduction de l'apport azoté en lactation, relation déjà entrevute par Pond (I973) et MaHax et Grifo (r975).

En conclusion, il ressort de cette étude qu'une réduction de l'apport azoté de lactation est possible chez la Truie primipare si l'on a augmenté l'apport protéique durant toute ou partie de la gestation, donc le niveau des réserves azotées qui sont alors utilisées pour pallier le déficit alimentaire de lactation. Cette utilisation des réserves n'est plus suffisante chez la 'Truie adulte pour assurer un gain de portée comparable à celui réalisé par l'animal recevant un apport plus large en protéines et la diminution de croissance de la portée est d'autant plus importante que 
le niveau des réserves de gestation est plus faible. Il apparaît donc judicieux chez la Truie d'adapter l'apport quantitatif et qualitatif de matières azotées aux besoins de l'animal durant chaque phase du cycle de reproduction.

Reçu pour publication en janvier $19 \pi 6$.

\author{
SUMMARY
}

\title{
CHRONOLOGY OF PROTEIN SUPPLY DURING THE REPRODUCTIVE CYCLE
}

This experiment was made during 2 reproductive cycles on 76 nulliparous sows of the Large White breed distributed into 3 groups only differring by the chronology of crude protein supply during the reproductive cycle. Group I (control) fed a diet with a low protein level during gestation (ro p. roo) and a high level during lactation ( 6 p. Ioo) was compared, on the one hand with group 3 receiving a diet with a constant protein level ( 13 p. Ioo) during the whole reproductive cycle and on the other, with group 2 in which the possibility of providing supplementary protein during late gestation (first Io p. Ioo) and then I 3 p. Ioo from day 75 of gestation) followed by a reduction of the lactation level ( 13 p. I oo) was examined. During each stage of the cycle, daily feed intake levels were identical for the three groups, i.e. $2 \mathrm{~kg}$ during gestation, 4.I7 (Ist cycle) and $+.8+$ (2nd cycle) during lactation, $2.5 \mathrm{~kg}$ between weaning and mating. The diet contained 3220 Kcal digestible energy per $\mathrm{kg}$ and crude protein in form of wheat replaced partially or not by soyabean. 50 meal (table 2 ).

During gestation, $30 \mathrm{p}$. Ioo increase in the intake of crude protein significantly enhanced the weight gain of the sows during the two cycles studied (table 3 ) : increase of gestation net gain (group 3 relatively to group I) was $32 \mathrm{p}$. Ioo (ist cycle) or $66 \mathrm{p}$. Ioo (2nd cycle). The growing consumption of crude protein during the last third of gestation (group 2) also increased the net weight gain of the animals : $36.5 \mathrm{~kg}$ versus 30.4 in group I (Ist cycle), 24 . I versus 19.6 in group I (2nd cycle).

Around day 82 of gestation, a study made on almost half of the animals from each group kept during two cycles in metabolism crates also showed an increase of the nitrogen balance at the highest dietary protein level : nitrogen retention (g per day) in groups $\mathbf{I}, 2,3$ was 8.3 , I5.5, I 2.5 respectively during the first cycle and 3.7, I0.8, I I.7 during the second cycle (tables 7 and 8$)$.

The protein supply during gestation did not affect either the litter size or the mean weight of the piglets at parturition (table 3). Likewise, the mean number of piglets (I 4.2) weaned by the sows having realized two lactations was not affected by the amount of crude protein ingested by the dams (table 4 ).

During the first lactation (35 days) the growth of the suckled piglets or the litter gain were comparable for the three groups (table 6), but lowering of the protein intake during lactation (groups 2 and 3) increased the weight loss in the dams: $17.7 \mathrm{~kg}$ (group 2) and $15.0 \mathrm{~kg}$ (group 3). versus $4.8 \mathrm{~kg}$ (group I).

During the second lactation, a non significant decrease in piglet weight at weaning was recorded in group $3(7.2 \mathrm{~kg}$ versus $7.5 \mathrm{~kg}$ in group $\mathrm{I})$ and notably in group $2(6.9 \mathrm{~kg})$. This retardation of piglet growth was mainly marked from day $2 \mathrm{I}$ of lactation. The litter gain was also lower in group $2\left(3^{8.8} \mathrm{~kg}\right)$ than in group $\mathbf{I}(49.15 \mathrm{~kg})$. That of group 3 was located between the two other groups $(45.15 \mathrm{~kg})$.

The milk composition of the sows was determined after 5 days of lactation (table 9). During the 2 cycles, the dry matter contents proved to be larger in group I (19.3 p. Ioo during the ist cycle and 18.45 during the 2 nd cycle) than in group 2 ( 18.0 and 17.9 p. Ioo, respectively) or group 3 (I8.25 and 17.4 p. Ioo respectively). Parallel to that the energy values of milk dry matter were higher in group I (6 $207 \mathrm{Kcal}$ during the rst cycle, $625 \mathrm{I} \mathrm{Kcal} \mathrm{during} \mathrm{the} \mathrm{2nd} \mathrm{cyclc)} \mathrm{than} \mathrm{in}$ group 2 (6 I 37 and 6 ooo, respectively) or group 3 (6 I $6 \mathrm{x}$ and $6025 \mathrm{Kcal}$, respectively).

Thus, it appears that reduction of the protein supply during lactation was possible in growing primiparous sows if this supply was enhanced during the whole gestation period or part of the latter, However, in adult sows the use of gestation protein reserves was not sufficient, under the experimental conditions, to obtain a litter gain comparable to that recorded in animals receiving a larger supply of protein during lactation. 


\section{RÉFÉRENCES BIBLIOGRAPHIQUES}

Agricultural Research Council (A.R.C.), I967. The nutrient requirements of farm livestork $n^{\circ} 3$ Pigs, A.R.C. London.

Baker D. H., Becker D. E., Jensen A. H., Harmon B. G., r97o. Reproductive performance and progeny development in swine as influenced by protein restriction during various portions of gestation. J. Anim. Sci., 31, 526-530.

De Geeter M. J., Hays V. W., Kratzer D. D., Cromwell G. L., I972. Reproductive performance of gilts fed diets low in protein during gestation and lactation. J. Anim. Sci., 35, 772-777.

Duḱe P. H., Rérat A., i975. Étude du besoin en lysine de la Truie gestante nullipare. Ann. Zontech., 24, 447-464.

Juée P. H., Pastuszewska B., Ë́tienne M., r975. Utilisation comparée de la féverole et du tourteau de soja par la Truie en lactation. II. Digestibilité et utilisation métabolique des acides aminés. Journées. Rech. porcine en France, I.N.R.A., I.T.P. éd., I2I-I28.

Elliott R. F., Vander Noot G. W., Gilbreath R. L, Fisher H., ig7r. Lffect of dietary protein level on composition changes in sow colostrum and milk. J. Anim. Sci., 32, II28-II37.

Elsley F. W. H., Bannerman M., Bathurst E. V. J., Bracewell A. G., Cunningham J. M, M., Dodswortir T. L., Dodds P. A., Forbes 'T. J., Laird R., I969. The effect of level of feed intake in pregnancy and in lactation upon the productivity of sows. Anim. Prod., 11, 225-24I.

Étienne M., Dute P. H., Pastuszewska B., 1975. Nitrogen balance in lactating sows fed on diets containing soybean oil meal or horsebean as a protein concentrate. Livestock Prod. Sci., 2, I47-I56.

Hawton J. D., Meade R. J., I97I. Influence of quantity and quality of protein fed the gravid female on reproductive performance and development of offspring in swine. J. Anim. Sci., 32, 88-95.

Holden P. J., Lucas E. W., Speer V. C., Hays V. W., ig68. Effect of protein level during pregnancy and lactation on reproductive performance in swine. J. Anim. Sci., 27, I587-I590.

MacPherson R. M., Elsley F. W. H., Smart R. I., I969. The influence of dietary protein intake during lactation on the reproductive performance of sows. Anim. Prod., 11, 443-45I.

Vahan D. C., Grifo A. P. Jr., 1975. Effects of dietary protein levels during lactation to first-litter: sows fed a fortified corn gestation diet. J. Anim. Sci., 41, I362-1367.

Mahan D. C., Mangan L. T., I975. Evaluation of various protein sequences on the nutritional carry over from gestation to lactation with first-litter sows. J. Nutr., 105, I29I-I 298.

Mahan D. C., Becker D. E., Jensen A. H., ig7r $a$. Effect of protein levels and Opacue-2 corn on sow and litter performance during the first and second lactation periods. J. A nim. Sci., 32, 470-475.

Mahan D. C., Becker D. E., Harmon B. G., Jensen A. H., ig7i $b$. Effect of protein levels and opaque- 2 corn on sow milk composition. J. Anim. Sci., 32, 482-486.

Miller G. M., Becker D. E., Jensen A. H., Harmon B. G., Norton H. W., ig69. F.ffect of protein intake on nitrogen retention by swine during late pregnancy. J. Anim. Sci., 28, 204-207.

National Research Council (N.R.C.), 1973. Nutrient requirements of swine. National Acadeny of Sciences. Washington.

O'Grady J. F., I97I. Level and source of protein in the diets of lactating sows. Ir. J. agric. Res., 10, I7-29.

Pond W. G., I973. Influence of maternal protein and energy nutrition during gestation on progeny performance in swine. J. anim. Sci., 36, I75-I82.

Pond W. G., Wagner W. C., Dunn J. A., Walker E. F., I968. Reproduction and early postnatal growth of progeny in swine fed a protein-free diet during gestation. J. Nutr., 94, 309-3I6.

Rérat A., Dú́e P. H., I975. Ernährung und Reproducktion der Sau. Übers. Tierernährg., 3, IOI-I4I U. 249-276.

Rippel R. H., Rasmussen O. G., Jensen A. H., Norton H. W., Becker D. E., I965. liffect of level and source of protein on reproductive performance of swine. J. anim. Sci., 24, 203-208.

Rombauts P., I962. Évolution de l'anabolisme gravidique chez la Truie en fonction de l'âge de l'animal. Ann. Zootech., 11, 39-5 I.

Salmon-Legagneur E., I965. Quelques aspects des relations nutritionnelles entre la grestation et la lactation chez la Truie. Ann. Zootech., 14, no H.S., 1-r37.

Salmon-Legagneur E., Legault C., Aumaitre A., ig66. Relations entre les variations pondérales de la Truie en reproduction et les performances d'élevage. Ann. Zootech., 15, 215-229. 